Research Article

\title{
Buckling Stability Analysis for Piles in the Slope Foundation Based on Cusp Catastrophe Theory
}

\author{
Rui Zhang ${ }^{D}$, Chuanxun Li, and Dandan Jin \\ Faculty of Civil Engineering and Mechanics, Jiangsu University, Zhenjiang 212013, China \\ Correspondence should be addressed to Rui Zhang; zhangrui@ujs.edu.cn
}

Received 2 February 2021; Revised 5 May 2021; Accepted 12 June 2021; Published 21 June 2021

Academic Editor: Jose Renato de Sousa

Copyright (c) 2021 Rui Zhang et al. This is an open access article distributed under the Creative Commons Attribution License, which permits unrestricted use, distribution, and reproduction in any medium, provided the original work is properly cited.

\begin{abstract}
The aim of this paper is to analyze the buckling stability problem for piles in the slope foundation based on cusp catastrophe theory. Formulation of critical buckling load of piles in the slope foundation is obtained. The influential factors of slope angle, distribution of landslide thrust behind the pile, pile-embedded ratio, pile constraints, pile-side friction, pile-side soil resistance, and pile socketed ratio upon buckling stability characteristic for piles in the slope foundation are examined. The results reveal that when pile diameter remains unchanged, critical buckling load increases with the increase of pile length when pile-embedded ratio reaches $60 \%$. When pile length remains unchanged, critical buckling load increases with the increase of pile diameter. Critical buckling load with the assumption of nonlinear horizontal elastic resistance of pile-side soil in the paper is more close to the value based on horizontal elastic resistance of pile-side soil suggested in the code. When slope angle increases, decreased extent of buckling critical load for piles 30-60 m in length is more obvious than the piles which are $10-30 \mathrm{~m}$ in length. Strengthening of pile constraints and increase of pile-embedded ratio and socketed ratio are helpful to pile critical buckling load increase. The influential factors of pile-side friction and landslide thrust behind the pile upon pile critical buckling load are tiny and can be neglected.
\end{abstract}

\section{Introduction}

During the $14^{\text {th }}$ Five-Year Plan Period, the pace of construction and upgrading will be promoted continuously in China. Meanwhile, as a mountainous country, mountainous and hilly areas cover two-thirds of the total land area. Therefore, with the continuous impetus of national infrastructure, as well as increasing piles of civil buildings, roads and bridges will be located in slope foundation. Piles in slope foundation are influenced by asymmetric pile-soil interaction, slope sliding force, and nonlinear pile-side soil resistance, whose buckling stability research is very vital. It is of important theoretical and practical significance to make deep research on buckling stability for piles in slope foundation [1].

Extensive studies with different approaches have been performed by many scholars on piles buckling stability analysis. Based on three-dimensional finite element method, Kurian and Srinivas [2] discussed bearing capacity for tapered piles and found that ultimate load-carrying capacities of tapered piles were higher than those of uniform piles by $10 \%$. Budkowska and Szymczak [3] utilized energy method to analyze buckling stability for partially embedded pile and revealed that the skin friction could be neglected if the ratio of the embedded length of the pile to its total length was less than 0.6. With dimensionless analytical method, Heelis et al. [4] made research into buckling stability for fully and partially embedded pile. Lee et al. [5] designed model experiment to estimate axial bearing capacity for tapered piles. The proposed method was valid for piles in sands of medium to dense conditions. Based on variation theory, Ren and Wang [6] discussed vertical bearing capacity for cement soil reinforced piles and predicted the performance of single JPP pile. Zou and Zhao [7] employed difference method to analyze axial loading performance for superlong piles on bedrock in soft soil and got the conclusion that when $l / d$ reaches a certain value, regardless of whether the pile toe is socketed in rock or not, the tip resistance will not be exerted. With cavity expansion theory and model experiment, Manandhar and 
Yasufuku [8] discussed vertical bearing capacity for tapered piles in sand and discovered that the pile load-settlement curves can easily be determined with the aid of the simple fundamental properties of soils with the closed-form solution. By means of numerical analysis method, Kong et al. [9] made research of bearing capacity for belled wedge pile and drew the conclusion that belled wedge pile can improve vertical load-carrying capacities under both compression and uplift loads and reduce the amount of negative skin friction developed. Bhattacharya and Goda [10] formulated probabilistic method to analyze buckling stability characteristic for axially loaded piles in liquefiable soils and found that boundary condition of the pile was essential to pile buckling stability. Nadeem et al. [11] adopted finite element method on the research of nonlinear stability for slender defect piles. The analyses' results showed that the load-carrying capacity of the piles decreased with increasing pile curvature and increasing slenderness ratio of the initially bent piles. With the help of energy method, Zhao et al. [12] carried out buckling stability research on bridge piles in steep slopes and put forward the following conclusions: (1) When pile-embedded ratio was $<0.61$, buckling point was in the free section of the pile. (2) Pile critical buckling load increased with increasing pile socketed ratio. When socketed ratio reached a certain value, its increasing effect to critical buckling load was exerted. Yin et al. [13] employed similarity theory in the indoor model experiment to discuss vertical bearing capacity and buckling stability for piles in slope foundation and concluded that the bigger the slope angle or free section of the pile, the more decrease of critical buckling load. Lu and Zhao. [14] adopted energy method to analyze calculated length for buckling stability of steel pipe pile and obtained the equations on calculated length of piles in uniform foundation and layered foundation. Lee et al. [15] applied Runge-Kutta method and Regula-Falsi method on the buckling stability research for tapered piles. The results showed that the proposed model provided good predictions of pile buckling response and can determine an optimal taper ratio such that the pile is capable of carrying a maximum flexural buckling load.

Cusp catastrophe theory [16] is a branch on discontinuity, which has developed greatly during the last 30 years and had a wide range of applications in pile ultimate bearing capacity prediction [17]. Some scholars have studied the buckling stability of piles based on cusp catastrophe theory. With static load test, Zhang and Cui [18] derived computing formula on ultimate vertical bearing capacity for a single end bearing pile and found that pile displacement value of instability point could be calculated out with parabolic load-displacement curve. Chen et al. [19] discussed buckling stability for Y-shape piles and established cusp catastrophe model for piles whose tips were socketed and tops were free; critical buckling loads of Y-shape piles were higher than those of uniform piles by $30 \%$. Li et al. [20] performed buckling stability analysis for tapered piles considering self-weight influence and concluded that self-weight of piles had a great influence on critical buckling load for tapered piles. When embedded ratio was less than $40 \%$ of pile length, larger critical buckling load would be obtained, which would cause unsafe bearing capacity designed according to the strength of pile body.

Based on the analysis of the existing literature, there are still some challenges on buckling stability analysis. (1) Cusp catastrophe theory is seldom adopted in the existing articles for in-slope pile buckling stability analysis. (2) Potential energy function is not complete, which may lead to incomprehensive influential factors discussion on pile buckling stability analysis.

The objective of this study is to analyze buckling stability for piles in slope foundation based on cusp catastrophe theory. The paper establishes total potential energy equation with assumptions of nonlinear distributed horizontal elastic resistance of pile-side soil and landslide thrust behind pile. Furthermore, the paper discusses influences of slope angle, landslide thrust distribution behind pile, pile-embedded ratio, pile constraints, pile-side soil friction, pile-side soil resistance, and pile socketed ration upon buckling stability for piles in slope foundation.

\section{Cusp Catastrophe Theory}

Potential function of cusp catastrophe model is defined as [16]

$$
V(x)=\frac{1}{4} x^{4}+\frac{1}{2} u x^{2}+v x,
$$

where $x$ is the system state variable and $u$ and $v$ are system control variables.

Making the derivation of potential function $V(x)$ zero, we can get equilibrium surface of cusp catastrophe model:

$$
V^{\prime}(x)=x^{3}+u x+v=0
$$

This surface is called catastrophe manifold, which indicates condition of system equilibrium state. The corresponding point is called critical or extreme point in mathematics.

System singular point sets satisfy equation (2) and the second derivative of potential function $V^{\prime \prime}(x)=0$; that is,

$$
V^{\prime \prime}(x)=3 x^{2}+u=0 .
$$

Eliminate $x$ through the combination of equations (2) and (3), and parametric plane equation is obtained as

$$
D=4 u^{3}+27 v^{2}=0 .
$$

Equation (4) is called system bifurcation sets, which denotes projection on the plane determined by control variables $u$ and $v$ of singular point sets. When system control variables satisfy equation (4), the system is in critical equilibrium state and will jump to a stable equilibrium state finally to achieve system catastrophe.

Curve $D$ is called bifurcation sets, whose discriminant is written as $D=4 u^{3}+27 v^{2}$.

When $D>0$, the system is in a stable state. When $D<0$, the system is in an unstable state. Catastrophe occurs when 
$u<0$ and $D=0$. In this state, there are three real roots for equation (4).

\section{Computation Model and Basic Assumptions}

Assuming that $\mathrm{L}$ is the total pile length, it can be divided into three sections: socketed section $l_{1}$, slope influenced section $l_{2}$, and free section $l$; that is, $L=l_{1}+l_{2}+l$. Vertical load on pile top is $P$. Pile embedded depth is $h: h=l_{1}+l_{2}$. The coordinate origin $o$ is at the center of pile bottom. Simplified computation model for buckling stability analysis of an in-slope pile is shown in Figure 1.

Pile bottom is a fixed constraint, pile top is free, $\alpha$ is the slope angle, $d$ is the pile diameter, $Q(x)$ is the landslide thrust behind the pile, $l_{2}=4 d \tan \alpha$ [13], and $\tau$ is the ultimate friction strength between pile and soil.

Basic assumptions in the computation are listed as follows:

(1) Ultimate friction strength between pile and soil $\tau$ is a constant.

(2) Horizontal elastic resistance of pile-side soil $q(x, y)$ is nonlinearly distributed within the range of embedded depth of pile, whose expression is regarded as follows [13]:

$$
q(x, y)= \begin{cases}m\left(h-l_{2}-x\right) b_{0} y & 0 \leq x \leq l_{1}, \\ m \frac{(h-x)^{2}}{l_{2}} b_{0} y & l_{1} \leq x \leq h,\end{cases}
$$

where $m$ is proportional coefficient of horizontal elastic resistance of pile-side soil. $b_{0}$ is calculated width of piles. For circular piles, $b_{0}=0.9(1.5 d+0.5)$, when $d<1.0 \mathrm{~m} . b_{0}=0.9$ $(d+1)$, when $d \geq 1.0 \mathrm{~m}$ [20]. Generalized expression of landslide thrust behind pile $Q(x)$ is regarded as [21]

$$
Q(x)=a_{1} x^{2}+b_{1} x+c_{1},
$$

where $a_{1}, b_{1}$, and $c_{1}$ are undetermined parameters related to the resultant force of landslide thrust $F$.

\section{Solution of Critical Buckling Load}

4.1. Establishment of Deflection Functions. According to the boundary conditions shown in Figure 1, the deflection function for a pile whose tip is socketed and top is free is established as $[12,20]$

$$
y=\sum_{n=1}^{\infty} C_{n}\left[1-\cos \left(\frac{2 n-1}{2 L} \pi x\right)\right],
$$

where $n$ is half-wave number of the deflection function. $C_{\mathrm{n}}$ is undetermined constant term. Under the small deformation assumption of pile-soil system, take $n=1$. Therefore, pile deflection function is expressed as

$$
y=C\left(1-\cos \frac{\pi x}{2 L}\right) .
$$

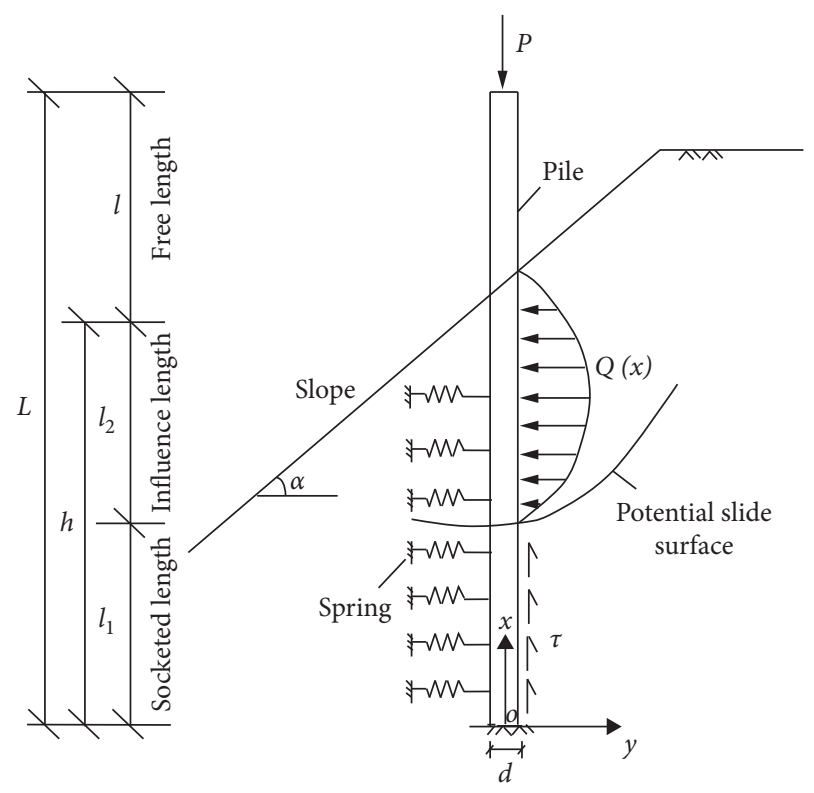

FIGURE 1: Simplified computation model for buckling stability analysis of an in-slope pile.

From equation (8), the first and second derivatives of pile deflection function are obtained as

$$
\begin{aligned}
& y^{\prime}=\frac{\pi C}{2 L} \sin \frac{\pi x}{2 L}, \\
& y^{\prime \prime}=\frac{\pi^{2} C}{4 L^{2}} \cos \frac{\pi x}{2 L} .
\end{aligned}
$$

4.2. Solution Based on Cusp Catastrophe Theory. The total potential energy equation of pile-soil system is established as follows $[12,20]$ :

$$
\Pi=U_{P}+U_{S}+V_{Q}+V_{P}+V_{G}+W_{f},
$$

where $U_{P}$ is the bending strain energy of the pile, $U_{S}$ is the elastic deformation energy of soil beside pile, $V_{Q}$ is the potential energy of landslide thrust behind the pile, $V_{P}$ is the potential energy of pile top load, $V_{G}$ is the potential energy of pile self-weight, and $W_{f}$ is the potential energy of pile-side friction embedded in soil. Substituting equations (8) (10), deduction of each potential energy item is listed as follows.

Bending strain energy of pile $U_{P}$ is as follows:

$$
\begin{aligned}
U_{P} & =\frac{\mathrm{EI}}{2} \int_{0}^{L} \frac{\left(\mathrm{d}^{2} y / \mathrm{d} x^{2}\right)^{2}}{\left[1+(\mathrm{d} y / \mathrm{d} x)^{2}\right]^{3}} \mathrm{~d} x \\
& =\frac{\mathrm{EI}}{2} \int_{0}^{L}\left(\frac{\mathrm{d}^{2} y}{\mathrm{~d} x^{2}}\right)^{2}\left[1-3\left(\frac{\mathrm{d} y}{\mathrm{~d} x}\right)^{2}\right] \mathrm{d} x \\
& =\frac{\pi^{4} \mathrm{EIC}^{2}}{32 L^{4}} \int_{0}^{L} \cos ^{2} \frac{\pi x}{2 L}\left[1-3 \frac{\pi^{2} C^{2}}{4 L^{2}} \sin ^{2} \frac{\pi x}{2 L}\right] \mathrm{d} x \\
& =\frac{\pi^{4} \mathrm{EI}}{64 L^{3}} C^{2}-\frac{3 \pi^{6} \mathrm{EI}}{1024 L^{5}} C^{4},
\end{aligned}
$$


where $E$ is the elastic modulus of the pile and $I$ is the moment of inertia of the pile section.
Deformation energy of soil beside pile $U_{S}$ is as follows:

$$
\begin{aligned}
U_{S}= & \frac{1}{2} \int_{0}^{h} q y \mathrm{~d} x \\
= & \frac{m b_{0}}{2} \int_{0}^{l_{1}}\left(h-l_{2}-x\right) C^{2}\left(1-\cos \frac{\pi x}{2 L}\right)^{2} \mathrm{~d} x+\frac{m b_{0}}{2 l_{2}} \int_{l_{1}}^{h}(h-x)^{2} C^{2}\left(1-\cos \frac{\pi x}{2 L}\right)^{2} \mathrm{~d} x \\
= & -\frac{m b_{0} C^{2}}{8 \pi^{2}}\left[\left(30 L^{2}-3 \pi^{2} l_{1}^{2}\right)+2 L^{2}\left(-16 \cos \frac{\pi l_{1}}{2 L}+\cos \frac{\pi l_{1}}{L}\right)\right] \\
& -\frac{m b_{0} C^{2}}{4 \pi^{3} l_{2}}\left[-\pi^{3} h^{3}+\pi^{3} l_{1}\left(3 h l_{2}+l_{1}^{2}\right)+32 \pi L^{2} l_{2}\left(\cos \frac{\pi l_{1}}{2 L}-\frac{1}{16} \cos \frac{\pi l_{1}}{L}\right)-64 L^{3}\left(\sin \frac{\pi h}{2 L}-\frac{1}{32} \sin \frac{\pi h}{L}\right)\right. \\
& \left.-8 L\left(-8 L^{2}+\pi^{2} l_{2}^{2}\right) \sin \frac{\pi l_{1}}{2 L}+L\left(-2 L^{2}+\pi^{2} l_{2}^{2}\right) \sin \frac{\pi l_{1}}{L}\right] \\
= & -\frac{m b_{0} C^{2}}{8 \pi^{2}}\left(A_{S 1}+32 L^{2} A_{S 2}-\frac{128 L^{3}}{\pi l_{2}} A_{S 3}-16 \pi L l_{2} A_{S 4}\right) \\
= & -\frac{m b_{0} A_{S}}{8 \pi^{2}} C^{2},
\end{aligned}
$$

where

$$
\begin{aligned}
& A_{S 1}=30 L^{2}-3 \pi^{2} l_{1}^{2}-\frac{2 \pi^{2} h^{3}}{l_{2}}+2 \pi^{2} l_{1}\left(3 h+\frac{l_{1}^{2}}{l_{2}}\right), \\
& A_{S 2}=\cos \frac{\pi l_{1}}{2 L}-\frac{1}{16} \cos \frac{\pi l_{1}}{L}, \\
& A_{S 3}=\left(\sin \frac{\pi h}{2 L}-\frac{1}{32} \sin \frac{\pi h}{L}\right)-\left(\sin \frac{\pi l_{1}}{2 L}-\frac{1}{32} \sin \frac{\pi l_{1}}{L}\right), \\
& A_{S 4}=\sin \frac{\pi l_{1}}{2 L}-\frac{1}{8} \sin \frac{\pi l_{1}}{L} .
\end{aligned}
$$

Potential energy of landslide thrust behind pile $V_{Q}$ is as follows: 


$$
\begin{aligned}
V_{Q}= & -\frac{1}{2} \int_{l_{1}}^{h} \frac{a_{1} x^{2}+b_{1} x+c_{1}}{l_{2}} y^{2} \mathrm{~d} x \\
= & -\frac{1}{2} \int_{l_{1}}^{h} \frac{C^{2}}{l_{2}}\left(a_{1} x^{2}+b_{1} x+c_{1}\right)\left(1-\cos \frac{\pi x}{2 L}\right)^{2} \mathrm{~d} x \\
= & -\frac{C^{2}}{8 \pi^{3} l_{2}}\left\{\pi^{3} h\left[6 c_{1}+h\left(3 b_{1}+2 a_{1} h\right)\right]-\pi^{3} l_{1}\left[6 c_{1}+l_{1}\left(3 b_{1}+2 a_{1} l_{1}\right)\right]-32 \pi L^{2}\left(b_{1}+2 a_{1} h\right)\left(\cos \frac{\pi h}{2 L}-\frac{1}{16} \cos \frac{\pi h}{L}\right)\right. \\
& -16 L\left[-8 a_{1} L^{2}+\pi^{2}\left(a_{1} h^{2}+b_{1} h+c_{1}\right)\right] \sin \frac{\pi h}{2 L} \\
& +2 L\left[-2 a_{1} L^{2}+\pi^{2}\left(a_{1} h^{2}+b_{1} h+c_{1}\right)\right] \sin \frac{\pi h}{L}+32 \pi L^{2}\left(b_{1}+2 a_{1} l_{1}\right)\left(\cos \frac{\pi l_{1}}{2 L}-\frac{1}{16} \cos \frac{\pi l_{1}}{L}\right) \\
& +16 L\left[-8 a_{1} L^{2}+\pi^{2}\left(a_{1} l_{1}^{2}+b_{1} l_{1}+c_{1}\right)\right] \sin \frac{\pi l_{1}}{2 L}-2 L\left[-2 a_{1} L^{2}+\pi^{2}\left(a_{1} l_{1}^{2}+b_{1} l_{1}+c_{1}\right)\right] \sin \frac{\pi l_{1}}{L} \\
= & -\frac{C^{2}}{8 \pi^{3} l_{2}}\left[B_{\mathrm{Q} 1}+B_{\mathrm{Q} 2}\left(\cos \frac{\pi h}{2 L}-\frac{1}{16} \cos \frac{\pi h}{L}\right)+B_{\mathrm{Q} 3}\left(\cos \frac{\pi l_{1}}{2 L}-\frac{1}{16} \cos \frac{\pi l_{1}}{L}\right)+B_{\mathrm{Q} 4}\left(\sin \frac{\pi h}{2 L}-\frac{1}{8} \sin \frac{\pi h}{L}\right)\right. \\
& \left.+B_{\mathrm{Q} 5}\left(\sin \frac{\pi l_{1}}{2 L}-\frac{1}{8} \sin \frac{\pi l_{1}}{L}\right)+128 a_{1} L^{3} B_{\mathrm{Q} 6}\right] \\
= & -\frac{B_{Q}}{8 \pi^{3} l_{2}} C^{2},
\end{aligned}
$$

where

$$
\begin{aligned}
& B_{Q 1}=\pi^{3} h\left[6 c_{1}+h\left(3 b_{1}+2 a_{1} h\right)\right]-\pi^{3} l_{1}\left[6 c_{1}+l_{1}\left(3 b_{1}+2 a_{1} l_{1}\right)\right] \\
& B_{Q 2}=-32 \pi L^{2}\left(b_{1}+2 a_{1} h\right), \\
& B_{Q 3}=32 \pi L^{2}\left(b_{1}+2 a_{1} l_{1}\right) \\
& B_{Q 4}=-16 \pi^{2} L\left(a_{1} h^{2}+b_{1} h+c_{1}\right), \\
& B_{Q 5}=16 \pi^{2} L\left(a_{1} l_{1}^{2}+b_{1} l_{1}+c_{1}\right), \\
& B_{Q 6}=\left(\sin \frac{\pi h}{2 L}-\frac{1}{32} \sin \frac{\pi h}{L}\right)-\left(\sin \frac{\pi l_{1}}{2 L}-\frac{1}{32} \sin \frac{\pi l_{1}}{L}\right) .
\end{aligned}
$$

Potential energy of pile top load $V_{P}$ is as follows:

$$
\begin{aligned}
V_{P} & =-P \int_{0}^{L}\left(\sqrt{1+\left(\frac{\mathrm{d} y}{\mathrm{~d} x}\right)^{2}}-1\right) \mathrm{d} x \\
& =-\frac{P}{2} \int_{0}^{L}\left[\left(\frac{\mathrm{d} y}{\mathrm{~d} x}\right)^{2}-\frac{1}{4}\left(\frac{\mathrm{d} y}{\mathrm{~d} x}\right)^{4}\right] \mathrm{d} x \\
& =-\frac{P}{2} \int_{0}^{L}\left(\frac{\pi^{2} C^{2}}{4 L^{2}} \sin ^{2} \frac{\pi x}{2 L}-\frac{\pi^{4} C^{4}}{64 L^{4}} \sin \frac{4}{2 L}\right) \mathrm{d} x \\
& =-\frac{\pi^{2} P}{16 L} C^{2}+\frac{3 \pi^{4} P}{1024 L^{3}} C^{4} .
\end{aligned}
$$

Potential energy of pile self-weight $V_{G}$ is as follows:

$$
\begin{aligned}
V_{G} & =-\gamma A \int_{0}^{L}(L-x)\left(\sqrt{1+\left(\frac{\mathrm{d} y}{\mathrm{~d} x}\right)^{2}}-1\right) \mathrm{d} x \\
& =-\frac{\gamma A}{2} \int_{0}^{L}(L-x)\left[\left(\frac{\mathrm{d} y}{\mathrm{~d} x}\right)^{2}-\frac{1}{4}\left(\frac{\mathrm{d} y}{\mathrm{~d} x}\right)^{4}\right] \mathrm{d} x \\
& =-\frac{\gamma A}{2} \int_{0}^{L}(L-x)\left(\frac{\pi^{2} C^{2}}{4 L^{2}} \sin ^{2} \frac{\pi x}{2 L}-\frac{\pi^{4} C^{4}}{64 L^{4}} \sin ^{4} \frac{\pi x}{2 L}\right) \mathrm{d} x \\
& =-\frac{\gamma A}{32}\left(\pi^{2}-4\right) C^{2}+\frac{\pi^{2} \gamma A\left(3 \pi^{2}-16\right)}{2048 L^{2}} C^{4},
\end{aligned}
$$

where $\gamma$ is the pile unit weight and $A$ is the pile section area.

Potential energy of pile-side friction $W_{f}$ is as follows:

$$
\begin{aligned}
W_{f}= & \tau U \int_{0}^{h}(h-x)\left(\sqrt{1+\left(\frac{\mathrm{d} y}{\mathrm{~d} x}\right)^{2}}-1\right) \mathrm{d} x \\
= & \frac{\tau U}{2} \int_{0}^{h}(h-x)\left[\left(\frac{\mathrm{d} y}{\mathrm{~d} x}\right)^{2}-\frac{1}{4}\left(\frac{\mathrm{d} y}{\mathrm{~d} x}\right)^{4}\right] \mathrm{d} x \\
= & \frac{\tau U}{2} \int_{0}^{h}(h-x)\left(\frac{\pi^{2} C^{2}}{4 L^{2}} \sin ^{2} \frac{\pi x}{2 L}-\frac{\pi^{4} C^{4}}{64 L^{4}} \sin \frac{4}{2 L}\right) \mathrm{d} x \\
= & \frac{\tau U C^{2}}{32 L^{2}}\left(-2 L^{2}+\pi^{2} h^{2}+2 L^{2} \cos \frac{\pi h}{L}\right) \\
& +\frac{\pi^{2} \tau U C^{4}}{4096 L^{4}}\left[15 L^{2}-6 \pi^{2} h^{2}+L^{2}\left(-16 \cos \frac{\pi h}{L}+\cos \frac{2 \pi h}{L}\right)\right] \\
= & \frac{\tau U C_{f}}{32 L^{2}} C^{2}+\frac{\pi^{2} \tau U \bar{C}_{f}}{4096 L^{4}} C^{4},
\end{aligned}
$$


where $U$ is the pile section circumference. The expressions of $C_{f}$ and $\mathrm{C}(-)_{\mathrm{f}}$ are written as

$$
\begin{aligned}
& C_{f}=\pi^{2} h^{2}-2 L^{2}\left(1-\cos \frac{\pi h}{L}\right), \\
& \bar{C}_{f}=15 L^{2}-6 \pi^{2} h^{2}-16 L^{2}\left(\cos \frac{\pi h}{L}-\frac{1}{16} \cos \frac{2 \pi h}{L}\right) .
\end{aligned}
$$

Substituting equations (12)-(19) into equation (11), the total potential energy equation of pile-soil system is expressed as

$$
\prod=\bar{a}_{0}+\bar{a}_{1} C+\bar{a}_{2} C^{2}+\bar{a}_{3} C^{3}+\bar{a}_{4} C^{4} .
$$

From equations (12)-(19), we can see that each expression of partial potential energy is the function of $C 2$ and $C 4$, so $\bar{a}_{0}=\bar{a}_{1}=\bar{a}_{3}=0$. The expressions of $\bar{a}_{2}$ and $\bar{a}_{4}$ can be written as

$$
\begin{aligned}
& \bar{a}_{2}=\frac{\pi^{4} E I}{64 L^{3}}-\frac{m b_{0} A_{S}}{8 \pi^{2}}-\frac{B_{Q}}{8 \pi^{3} l_{2}}-\frac{\pi^{2} P}{16 L}-\frac{\gamma A}{32}\left(\pi^{2}-4\right)+\frac{\tau U C_{f}}{32 L^{2}}, \\
& \bar{a}_{4}=-\frac{3 \pi^{6} E I}{1024 L^{5}}+\frac{3 \pi^{4} P}{1024 L^{3}}+\frac{\pi^{2} \gamma A\left(3 \pi^{2}-16\right)}{2048 L^{2}}+\frac{\pi^{2} \tau U \bar{C}_{f}}{4096 L^{4}} .
\end{aligned}
$$

Substituting variables of $C$, let $C=t\left(1 / 4 \bar{a}_{4}\right)^{1 / 4}, \bar{a}_{4}>0$, and the total potential energy equation (21) is expressed as

$$
\prod=\frac{1}{4} t^{4}+\frac{1}{2} u t^{2}+v t
$$

where $u=\bar{a}_{2} / \sqrt{\bar{a}_{4}}$ and $v=\bar{a}_{1} /\left(4 \bar{a}_{4}\right)^{1 / 4}=0$.

According to cusp catastrophe theory, the discriminant is written as

$$
D=4 u^{3}+27 v^{2}=4\left(\frac{\bar{a}_{2}}{\sqrt{\bar{a}_{4}}}\right)^{3} .
$$

When $D=0$, system catastrophe occurs. Therefore, it is assumed that

$$
D=4 u^{3}+27 v^{2}=4\left(\frac{\bar{a}_{2}}{\sqrt{\bar{a}_{4}}}\right)^{3}=0 .
$$

From equation (25), the expression of pile critical buckling load $P_{c r}$ is obtained as

$$
P_{c r}=\frac{\pi^{2} \mathrm{EI}}{4 L^{2}}-\frac{2 m b_{0} \mathrm{LA}_{S}}{\pi^{4}}-\frac{2 \mathrm{LB}_{Q}}{\pi^{5} l_{2}}-\frac{\gamma \mathrm{AL}}{2 \pi^{2}}\left(\pi^{2}-4\right)+\frac{\tau \mathrm{UC}_{f}}{2 \pi^{2} L} \text {. }
$$

\section{Correctness Verification of Critical Buckling Load Expression}

According to critical buckling load expression of equal section pile in level ground from [20], suppose that slope angle $\alpha=0$, and horizontal elastic resistance of pile-side soil $q(x, y)$ is adopted as $q(x, y)=m b_{0}(h-x) y$. In the meantime, the influences of ultimate friction strength between pile and soil $\tau$ and landslide thrust behind pile are neglected, and formula (26) can be written as follows:

$$
P_{c r}=\frac{\pi^{2} E I}{4 L^{2}}+\frac{2 m b_{0} L^{3}}{\pi^{4}}\left[-30+3 \pi^{2}\left(\frac{h}{L}\right)^{2}+2\left(16 \cos \frac{\pi h}{2 L}-\cos \frac{\pi h}{L}\right)\right]-\frac{\gamma \mathrm{AL}}{2 \pi^{2}}\left(\pi^{2}-4\right) .
$$

The derived critical buckling load expression of equal section pile in level ground from formula (26) is the same as that in [20].

Furthermore, considering pile self-weight zero and pileembedded depth $h=0$, expression (27) can be simplified as

$$
P_{c r}=\frac{\pi^{2} \mathrm{EI}}{4 L^{2}}
$$

According to expression (28), we obtain critical buckling load of ordinary compressed bar whose tip is socketed and top is free. Therefore, critical buckling load expression (26) for piles in slope foundation derived in the paper is reliable.

\section{Case Study}

The pile is in slope foundation, pile concrete strength grade is $C 25$, slope soil is soft plastic clay, and slope angle $\alpha$ is $30^{\circ}$. Pile-embedded ratio $h / L$ is 0.6 . Landslide thrust behind pile $Q(x)$ is assumed as triangular distribution. Composition of landslide thrust behind pile is $80 \mathrm{kN} / \mathrm{m}$. Other pile and soil parameters are obtained from the work of Zhao et al. [12] and listed in Table 1. Physical meanings of the input parameters are explained in the previous section.

6.1. Variation Analysis of Pile Buckling Load with Different Pile Lengths. Critical buckling load changes for piles in slope foundation with varying pile diameter and length are shown in Figure 2. Pile length ranges from $10 \mathrm{~m}$ to $60 \mathrm{~m}$. Pile diameter $d$ is $0.5 \mathrm{~m}, 0.75 \mathrm{~m}, 1.0 \mathrm{~m}, 1.5 \mathrm{~m}$, and $2.0 \mathrm{~m}$. Pileembedded ratio $h / L$ is 0.6 .

It can be seen from Figure 2 that when pile diameter $d$ remains unchanged, with the increase of pile length $L$, pile socketed length increases, and critical buckling load of piles in slope foundation increases accordingly. When pile length $L$ remains unchanged, with the increase of pile diameter $d$, the pile slenderness ratio $L / d$ decreases, and critical buckling load of piles in slope foundation increases accordingly.

6.2. Influence of Slope Angle. With slope angle $\alpha$ being $0^{\circ}, 10^{\circ}$, $20^{\circ}, 30^{\circ}, 40^{\circ}, 50^{\circ}$, and $60^{\circ}$, the influence of slope angle on 
TABLE 1: Material parameters for the pile and soil.

\begin{tabular}{lccc}
\hline$E(\mathrm{MPa})$ & $\gamma\left(\mathrm{kN} \bullet \mathrm{m}^{-3}\right)$ & $m\left(\mathrm{MN} \bullet \mathrm{m}^{-4}\right)$ & $\tau(\mathrm{kPa})$ \\
\hline $2.38 \times 10^{4}$ & 25 & 5.0 & 20 \\
\hline
\end{tabular}

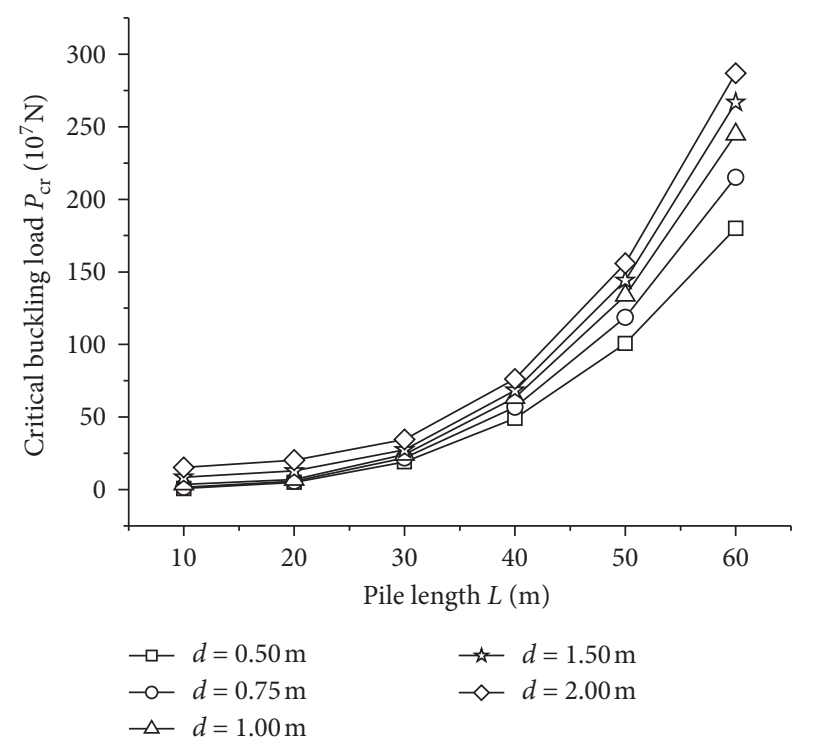

Figure 2: $P_{c r}-L$ curve with different pile lengths.

critical buckling load of piles in slope foundation is discussed. Critical buckling load change curves of piles in slope foundation with varying slope angle are shown in Figure 3.

It can be seen from Figure 3 that, compared with pile in level ground $\left(\alpha=0^{\circ}\right)$, in-slope pile critical buckling load decreases. For medium-long piles in the range of $10-20 \mathrm{~m}$, critical buckling load reduction is small when slope angle increases. For long piles in the range of $30-40 \mathrm{~m}$ and superlong piles over $50 \mathrm{~m}$, critical buckling load reduction is obvious when slope angle increases. Regardless of the pile length, critical buckling load decreases proportionally with the angle approximately. That is because when pile-embedded ratio is constant, socketed length $l_{1}$ decreases proportionally with slope angle approximately.

6.3. Influence of Landslide Thrust behind the Pile. According to landslide thrust distribution function table in [21], parabola distribution, linear distribution, trapezoidal distribution, triangular distribution, and no thrust distribution of landslide thrust are employed, and the influence of landslide thrust behind pile upon critical buckling load is discussed. Composition of landslide thrust behind pile is $80 \mathrm{kN} / \mathrm{m}$. Critical buckling load change curves of piles in the slope foundation with varying landslide thrust are listed in Table 2.

It can be seen from Table 2 that when landslide thrust behind pile is considered, critical buckling load of piles in slope foundation decreases with tiny reduction. Compared with critical buckling load without thrust distribution, the reduction is less than $0.5 \%$. Critical buckling load varies with tiny reduction with parabola, linear, trapezoidal, and triangular distribution. Therefore, for in-slope pile buckling stability analysis, influence of landslide thrust behind pile upon critical buckling load is tiny and can be neglected.

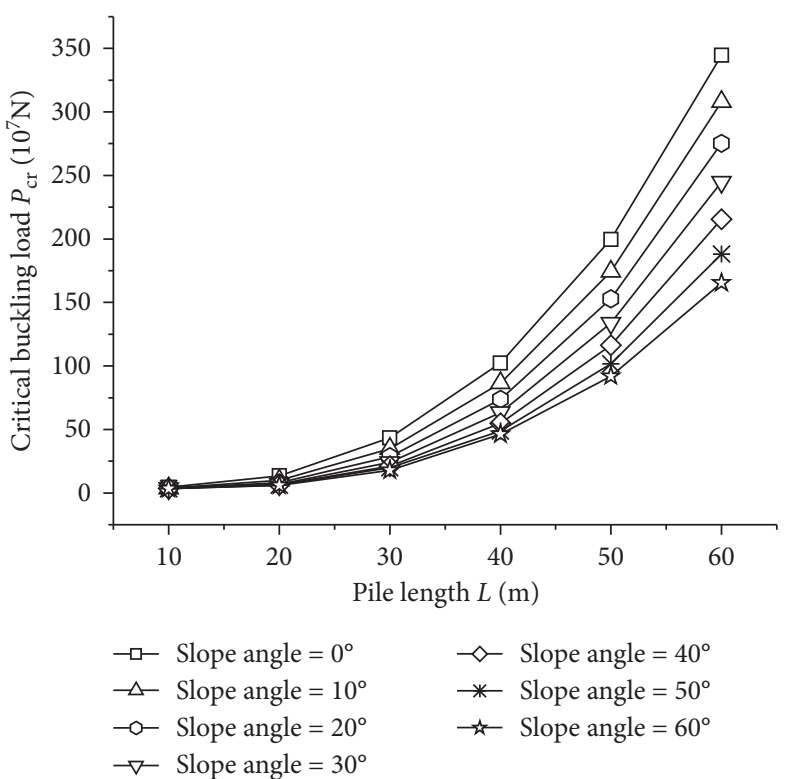

FIgURE 3: Critical buckling load with varying slope angle.

6.4. Influence of Pile-Embedded Ratio. Pile-embedded ratio $h / L$ is $0.15,0.20,0.30,0.40,0.50,0.60,0.70$, and 0.75 , and the influence of pile-embedded ratio upon critical buckling load is discussed. Critical buckling load change curves of piles in slope foundation with varying pile-embedded ratio are shown in Figure 4.

It can be seen from Figure 4 that when pile-embedded ratio $h / L \leq 0.20$, critical buckling load of piles in slope foundation decreases gradually with the increase of pile length. At this moment, critical buckling point is in the free section of the pile. When pile-embedded ratio $h / L$ is in the range of 0.30 to 0.50 , critical buckling load of piles in slope foundation decreases first and increases afterwards with the increase of pile length. Critical buckling point transits from free section to influenced section of piles. When pile-embedded ratio $h / L \geq 0.60$, critical buckling load of piles in slope foundation increases with the increase of pile length. Therefore, pile-embedded ratio $h / L$ should reach $60 \%$ for piles in slope foundation.

6.5. Influence of Pile Constraints. Constraint types of pile tip and top include free, elastic embedded, articulated, and fixed. Suppose that pile tip constraint is fixed and pile top constraint is one of the above four constraints. Combine the constraint conditions of pile tip and pile top afterwards. With the assumption of small deformation of pile-soil system, influence of pile constraints upon critical buckling load of piles in slope foundation is discussed. The corresponding pile deflection functions are listed in Table 3 [22].

Critical buckling load change curves of piles in slope foundation with varying pile top constraints are shown in Figure 5 .

It can be seen from Figure 5 that critical buckling load is of the minimum value when pile top is free. Piles are most prone to buckling instability. Critical buckling load is of the maximum value when pile top is fixed. Piles are the least 
TABLE 2: Critical buckling load for piles in the slope foundation with varying landslide thrust distribution (MN).

\begin{tabular}{lccccc}
\hline$L(\mathrm{~m})$ & Parabola distribution & Linear distribution & Trapezoidal distribution & Triangular distribution & No thrust distribution \\
\hline 10 & 36.108 & 36.099 & 36.112 & 36.114 & 36.153 \\
20 & 68.221 & 68.208 & 68.227 & 68.229 & 68.363 \\
30 & 240.959 & 240.946 & 240.965 & 240.968 & 241.206 \\
40 & 632.638 & 632.627 & 632.646 & 632.648 & 632.996 \\
50 & 1336.587 & 1336.578 & 1336.600 & 1336.602 & 1336.991 \\
60 & 2447.879 & 2447.877 & 2447.899 & 2447.901 & 2448.464 \\
\hline
\end{tabular}

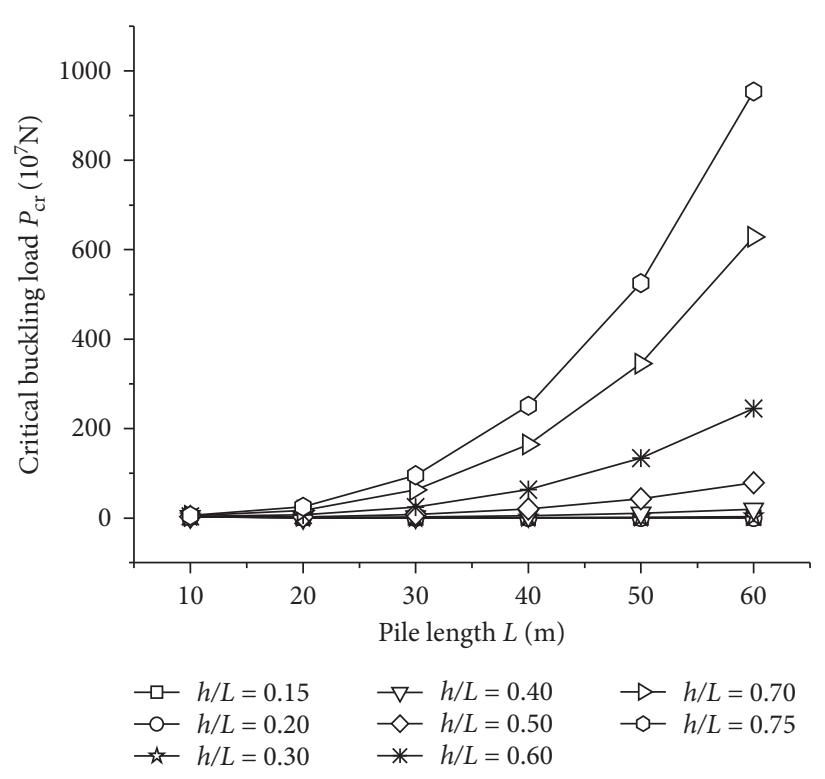

(a)

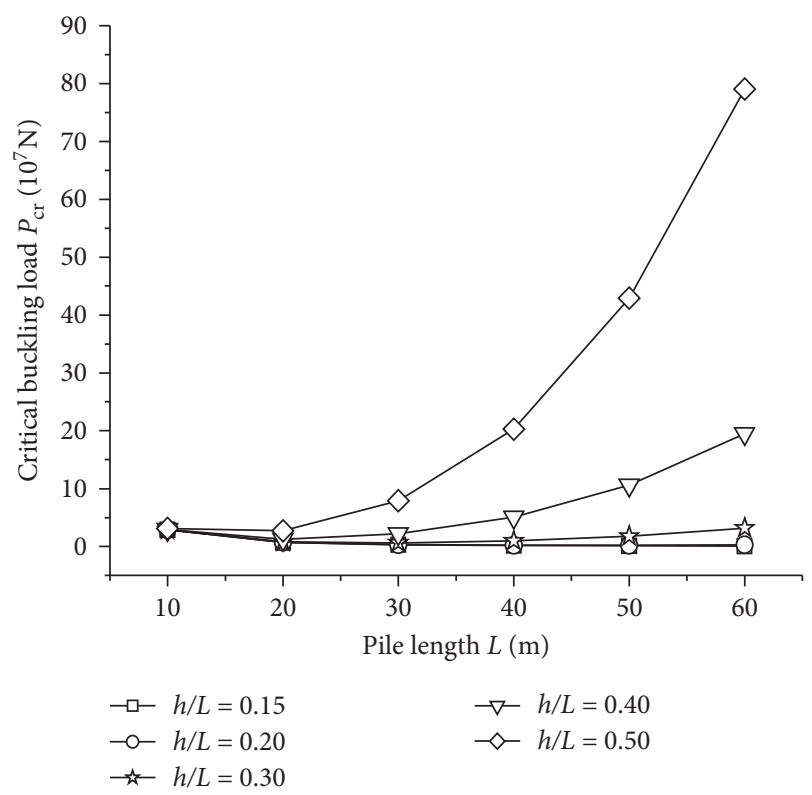

(b)

Figure 4: Critical buckling load with varying pile-embedded ratio. (a) Global graph. (b) Partial enlarged graph.

prone to buckling instability. Critical buckling load is between the previous values when pile top is elastic embedded or articulated. Critical buckling load with articulated pile top is a little more than the value with elastic embedded pile top. Therefore, when pile tip is fixed, critical buckling load of piles in slope foundation can be improved effectively with strengthening of pile top constraints.

6.6. Influence of Pile-Side Friction. Let ultimate friction strength between pile and soil $\tau$ be 0,30 , and $60 \mathrm{kPa}$. Pileembedded ratio $h / L$ is 0.6 . The influence of pile-side friction upon critical buckling load is discussed. Critical buckling loads with different ultimate friction strength between pile and soil and pile length are shown in Table 4 .

It can be seen from Table 4 that when pile-side friction is considered, critical buckling load of piles in slope foundation increases. But the extent of increase is less than $0.5 \%$. Therefore, the influence of pile-side friction upon critical buckling load can be neglected.

6.7. Influence of Horizontal Elastic Resistance of Pile-Side Soil. The typical methods to calculate horizontal elastic resistance of pile-side soil include Constant method, $m$ method, and $c$ method. Among them, $m$ method is the method adopted in
TABle 3: Deflection functions under different pile constraints.

\begin{tabular}{lc}
\hline Pile top constraint & Deflection function \\
\hline Free & $y=C(1-\cos \pi x / 2 L)$ \\
Elastic embedded & $y=C(1-\cos \pi x / L)$ \\
Articulated & $y=C(\cos 3 \pi x / 2 L-\cos \pi x / 2 L)$ \\
Fixed & $y=C(1-\cos 2 \pi x / L)$ \\
\hline
\end{tabular}

the current national code [23] to calculate foundation counterforce. At present, some scholars combine $m$ method and $c$ method with Constant method to calculate pile-side soil resistance. In this section, critical buckling load of piles in slope foundation is obtained with different pile-side soil resistance computation methods. The expressions assumed in the above methods are listed in Table 5 (the expressions assumed in the paper are given in equation (5)).

$q(x, y)$ can be expressed as

$$
q(x, y)=k(x) y,
$$

where $K(x)$ is pile-side soil resistance coefficient. Therefore, pile-side soil resistance distribution along pile length is different for the above methods. Pile-side soil resistance is linear distribution along pile length for $m$ method. Pile-side soil resistance is 0.5 power exponential distribution for $c$ method. For $m$-Constant method and $c$-Constant method, pile-side soil resistance is constant when $0 \leq x<l_{1}$ and linear 


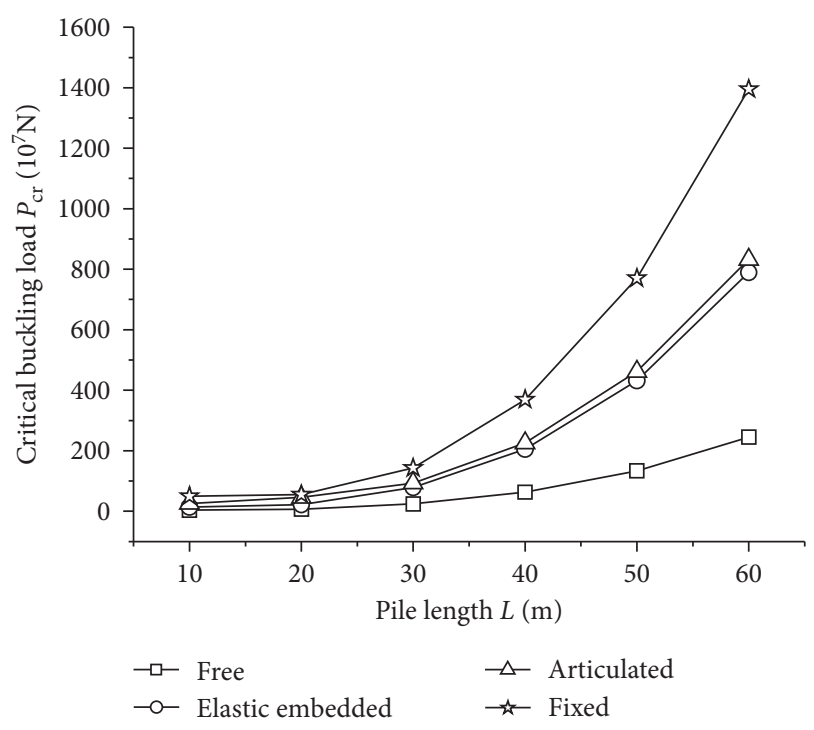

FIGURE 5: Critical buckling load with varying pile top constraints.

TABLE 4: Critical buckling load for piles in the slope foundation with varying limit friction strength $(\mathrm{MN})$.

\begin{tabular}{lccc}
\hline$L(\mathrm{~m})$ & $\tau=0 \mathrm{kPa}$ & $\tau=30 \mathrm{kPa}$ & $\tau=60 \mathrm{kPa}$ \\
\hline 10 & 36.084 & 36.129 & 36.174 \\
20 & 68.169 & 68.259 & 68.348 \\
30 & 240.879 & 241.013 & 241.147 \\
40 & 632.531 & 632.710 & 632.888 \\
50 & 1336.45 & 1336.67 & 1336.90 \\
60 & 2447.71 & 2447.98 & 2448.25 \\
\hline
\end{tabular}

or 0.5 power exponential distribution when $l_{1} \leq x \leq h$. As shown in expression (5), for pile-side soil resistance suggested in the paper, it is linear distribution when $0 \leq x<l_{1}$ and square exponential distribution when $l_{1} \leq x \leq h$.

Critical buckling load change curves of piles with varying pile-side soil resistance computation methods are shown in Figure 6.

It can be seen from Figure 6 that, for medium-long piles in the range of $10 \mathrm{~m}-20 \mathrm{~m}$, difference of critical buckling load with varying pile-side soil resistance computation methods is not wide. For long piles in the range of 30-40 m and superlong piles over $50 \mathrm{~m}$, difference of critical buckling load with varying pile-side soil resistance computation methods appears gradually. Critical buckling load obtained with pile-side soil resistance computation method suggested in the paper is close to the value with $m$ method adopted in the current national code. Therefore, pile-side soil resistance computation method suggested in the paper is of certain usability.

6.8. Influence of Pile Socketed Ratio. For piles in slope foundation with different lengths, the influence of pile socketed ratio $l_{1} / L$ upon critical buckling load is discussed through increase of pile socketed ratio. Critical buckling load change curves of piles in slope foundation with different lengths and pile socketed ratio are shown in Figure 7.
TABle 5: Pile-side soil resistance expressions.

\begin{tabular}{lcc}
\hline Method name & $0 \leq x<l 1$ & $q(x, y)$ \\
& \multicolumn{2}{c}{$l 1 \leq x \leq h$} \\
\hline$m$ method & $m b_{0}(h-x) y$ \\
$c$ method & $m b_{0}(h-x)^{0.5} y$ \\
$m$-constant method & $m b_{0}\left(h-l_{1}\right) y_{0} \quad m b_{0}(h-x) y$ \\
$c$-constant method & $m b_{0}\left(h-l_{1}\right)^{0.5} y$ & $m b_{0}(h-x)^{0.5} y$ \\
\hline
\end{tabular}

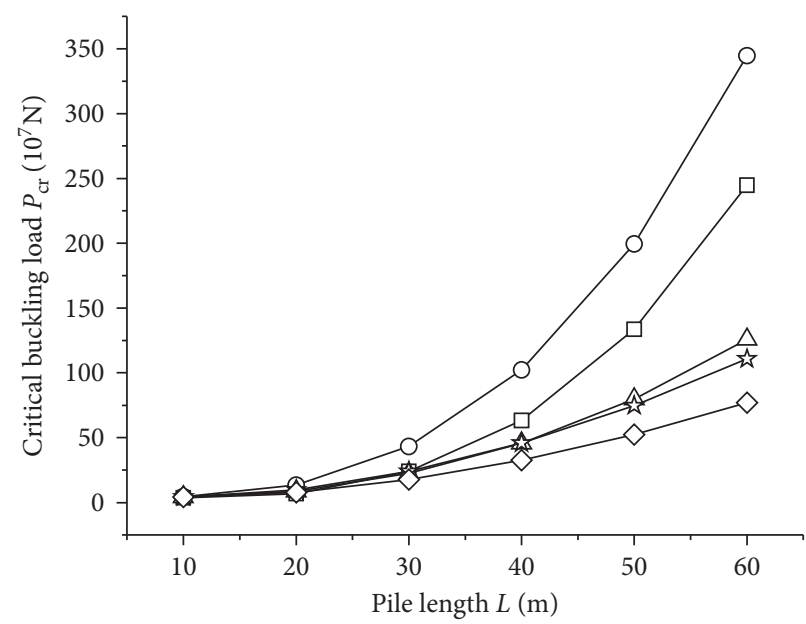

$$
\begin{aligned}
& \rightarrow-\text { Method in the paper } \rightarrow-m \text {-constant method } \\
& \neg-m \text { method } \\
& -\backsim c \text { method }
\end{aligned}
$$

Figure 6: Critical buckling load with varying pile-side soil resistance computation methods.

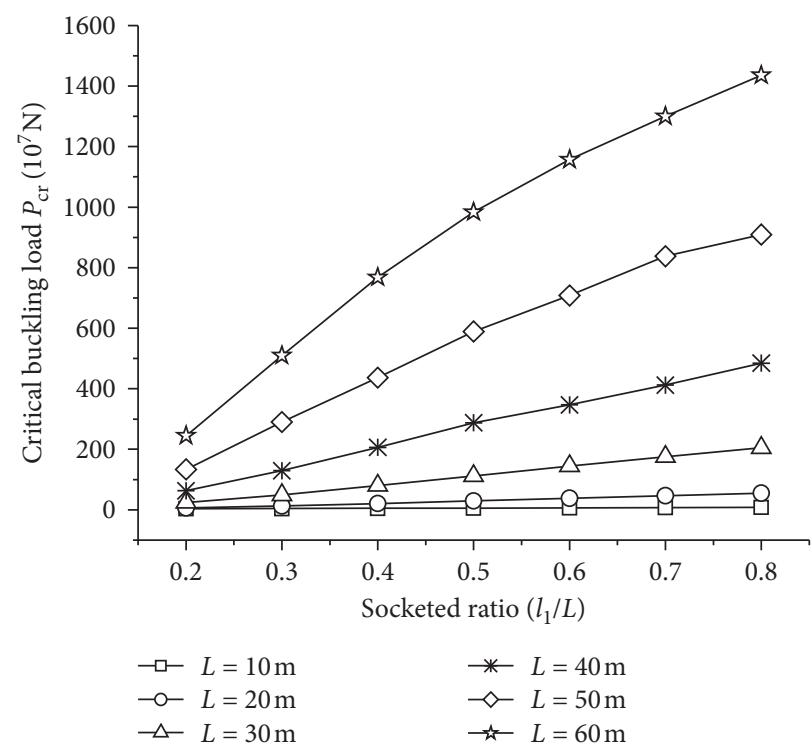

FIGURE 7: Critical buckling load with varying pile socketed ratio.

It can be seen from Figure 7 that critical buckling load of piles in slope foundation increases with the increase of pile socketed ratio. That is to say, appropriate increase of pile socketed length is helpful to increase pile critical buckling load. Furthermore, the more of pile length, the more of increase latitude of pile critical buckling load. There is 
especially obvious increase latitude for superlong piles over $50 \mathrm{~m}$. Critical buckling load change curves will become flat when pile socketed ratio reaches a certain value. That is to say, there is a critical fixed depth [12]. Critical buckling load change curves of medium-long piles in the range of $10 \mathrm{~m}-20 \mathrm{~m}$ tend to be flat earlier.

\section{Conclusions}

Based on cusp catastrophe theory, buckling stability parametric study for piles in slope foundation was made. The expression of pile critical buckling load is derived. The influences of slope angle, landslide thrust distribution behind pile, pile-embedded ratio, pile constraints, pile-side soil friction, pile-side soil resistance, and pile socketed ration upon buckling stability for piles in slope foundation are discussed. The key observations of the present work are as follows:

(1) When pile diameter remains unchanged, critical buckling load of piles in slope foundation increases with the increase of pile length if the $h / L$ ratio is constant. When pile length remains unchanged, with the increase of pile diameter, the pile slenderness ratio decreases, and critical buckling load of piles in slope foundation increases. We get the result making pile-embedded ratio $60 \%$.

(2) Compared with pile in level ground, critical buckling load of piles in slope foundation decreases. For long piles in the range of $30-40 \mathrm{~m}$ and superlong piles over $50 \mathrm{~m}$, the extent of reduction of critical buckling load is obvious when the slope angle increases.

(3) Critical buckling load of piles in slope foundation is influenced by pile constraints. Suppose that pile tip constraint is fixed. Critical buckling load is of the minimum value when the pile top is free. Critical buckling load is of the maximum value when the pile top is fixed. Piles are the least prone to buckling instability. Therefore, critical buckling load of piles in slope foundation can be improved effectively with strengthening of pile constraints.

(4) Critical buckling load of piles in slope foundation is greatly influenced by pile-embedded ratio and pile socketed ratio. Pile-embedded ratio should reach $60 \%$ for piles in slope foundation. Critical buckling load of piles in slope foundation increases with the increase of pile socketed ratio. When socketed ratio reached certain value, its increasing effect to critical buckling load was exerted and critical buckling load change curves will become flat. Medium-long piles in the range of $10 \mathrm{~m}-20 \mathrm{~m}$ tend to reach critical socketed depth earlier.

(5) Critical buckling load of piles over $30 \mathrm{~m}$ is greatly influenced by pile-side soil resistance. Critical buckling load obtained with the pile-side soil resistance computation method suggested in the paper is close to the value with $m$ method adopted in the current national code. The influence of pile-side friction and landslide thrust behind pile upon critical buckling load of piles in slope foundation is tiny, and both of them can be neglected.

\section{Data Availability}

The data that support the findings of this study are available from the corresponding author upon reasonable request.

\section{Conflicts of Interest}

The authors declare that there are no conflicts of interest regarding the publication of this paper.

\section{Acknowledgments}

This research was funded by the National Natural Science Foundation of China (Grant no. 51708256), Jiangsu Province Postdoctoral Foundation (no. 1301067B), Open Innovation Foundation of Changjiang Institute of Survey, Planning, Design and Research (Grant no. CX2020K08), and Advanced Expert Foundation of Jiangsu University (Grant no. 09JDG058).

\section{References}

[1] H. Zhao, P.-B. Yin, and X.-B. Li, "Mechanical response of bridge piles in high-steep slopes and sensitivity study," Journal of Central South University of Central, vol. 22, no. 10, pp. 4043-4048, 2015.

[2] N. P. Kurian and M. S. Srinivas, "Studies on the behaviour of axially loaded tapered piles by the finite element method," International Journal for Numerical and Analytical Methods in Geomechanics, vol. 19, no. 12, pp. 869-888, 1995.

[3] B. B. Budkowska and C. Szymczak, "Partially embedded piles subjected to critical buckling load-sensitivity analysis," Computers \& Structures, vol. 61, no. 1, pp. 193-196, 1996.

[4] M. E. Heelis, M. N. Pavlović, and R. P. West, "The analytical prediction of the buckling loads of fully and partially embedded piles," Géotechnique, vol. 54, no. 6, pp. 363-373, 2004.

[5] J. Lee, K. Paik, D. Kim, and S. Hwang, "Estimation of axial load capacity for bored tapered piles using CPT results in sand," Journal of Geotechnical and Geoenvironmental Engineering, vol. 135, no. 9, pp. 1285-1294, 2009.

[6] L. W. Ren and G. Y. Wang, "Practical variational analysis on vertical behavior of jet grouting soil-cement-pile strengthened pile,” ASCE GSP, vol. 220, pp. 126-134, 2011.

[7] X.-J. Zou and M.-H. Zhao, "Axial bearing behavior of superlong piles in deep soft clay over stiff layers," Journal of Central South University, vol. 20, no. 7, pp. 2008-2016, 2013.

[8] S. Manandhar and N. Yasufuku, "Vertical bearing capacity of tapered piles in sands using cavity expansion theory," Soils and Foundations, vol. 53, no. 6, pp. 853-867, 2013.

[9] G. Q. Kong, Q. Yang, H. L. Liu, and R. Y. Liang, "Numerical study of a new belled wedge pile type under different loading modes," European Journal of Environmental and Civil Engineering, vol. 17, pp. 65-82, 2013.

[10] S. Bhattacharya and K. Goda, "Probabilistic buckling analysis of axially loaded piles in liquefiable soils," Soil Dynamics and Earthquake Engineering, vol. 45, pp. 13-24, 2013.

[11] M. Nadeem, T. Chakraborty, and V. Matsagar, "Nonlinear buckling analysis of slender piles with geometric 
imperfections," Journal of Geotechnical and Geoenvironmental Engineering, vol. 141, Article ID 6014014, 2015.

[12] M. H. Zhao, W. H. Liu, P. B. Yin, C. W. Yang, and H. Zhao, "Buckling analysis of bridge piles in steep slopes based on energy method," Journal of Central South University (Science and Technology), vol. 47, no. 2, pp. 586-592, 2016.

[13] P. B. Yin, Y. Yang, W. He, X. X. Liu, and H. Zhao, "Analysis of critical buckling loads of piles considering slope effect," Rock and Soil Mechanics, vol. 38, no. 9, pp. 2662-2669, 2017.

[14] W. Lu and D. Zhao, "Analysis on calculated length for buckling stability of steel pipe pile based on energy method," The Open Civil Engineering Journal, vol. 11, no. 1, pp. 167-175, 2017.

[15] J. K. Lee, S. Jeong, and Y. Kim, "Buckling of tapered friction piles in inhomogeneous soil," Computers and Geotechnics, vol. 97, pp. 1-6, 2018.

[16] R. Thom, Structural Stability and Morphogenesis, W A Benjamin, Washington, NY, USA, 1978.

[17] K. Yang, T. Wang, and Z. Ma, "Application of cusp catastrophe theory to reliability analysis of slopes in open-pit mines," Mining Science and Technology (China), vol. 20, no. 1, pp. 71-75, 2010.

[18] Y. F. Zhang and S. Q. Cui, "Analysis on vertical bearing capacity of end-bearing pile foundation based on the catastrophe theory," Rock and Soil Mechanics, vol. 28, pp. 901-904, 2007.

[19] Y. H. Chen, X. Q. Wang, and H. L. Liu, "Buckling critical load analysis of $Y$ style vibro-pile based on cusp catastrophe theory," Engineering Mechanics, vol. 26, no. 4, pp. 119-127, 2009.

[20] C. X. Li, X. Z. Liu, and W. B. Wu, "Buckling analysis for tapered pile considering self-weight of pile," Journal of Southwest Jiaotong University, vol. 52, no. 6, pp. 1130-1138, 2016.

[21] Z. H. Dai, "Study on distribution laws of landslide-thrust and resistance of sliding mass acting on antislide piles," Chinese Journal of Rock Mechanics and Engineering, vol. 21, no. 4, pp. 517-521, 2002.

[22] H. F. Shan, T. D. Xia, F. Yu, and J. H. Hu, "Critical buckling capacity of piles with different pile-head constraints for excavation beneath existing foundation," Chinese Journal of Geotechnical Engineering, vol. 39, pp. 49-52, 2017.

[23] The Professional Standards Compilation Group of People's Republic of China, JGJ 94-2008. Technical Code for Building Pile Foundations, China Architecture and Building Press, Beijing, China, 2008. 